\title{
Der innerpsychische Konflikt Individuation vs. Abhängigkeit im Coaching: Zentrale Ergebnisse einer qualitativen Studie zur Praxis erfahrener Coaches
}

\author{
Claudia Halberstadt
}

Eingegangen: 10. August 2021 / Angenommen: 17. September 2021 / Online publiziert: 9. November 2021

(C) Der/die Autor(en) 2021

Zusammenfassung Die unbewusste Dynamik von innerpsychischen Konflikten ist ein wichtiger Inhalt des Erlebens und Verhaltens von Menschen und hat vermutlich einen Einfluss in Coachingprozessen. Diese Studie fokussiert den Grundkonflikt „Individuation vs. Abhängigkeit“" in Einzelcoachings. In einer kleinen qualitativen Feldstudie $(n=13)$ wurden erfahrene Coaching-Expert/innen zu subjektiven Theorien und präferierten Arbeitskonzepten im Handling dieses Konflikts im Einzelcoaching befragt. Die Inhaltsanalyse zeigt Strategien zum Handling damit auf. Im Artikel geht es primär um die Darstellung, Interpretation und Diskussion eines zentralen Forschungsergebnisses, nämlich der Bedeutung der Coachingbeziehung.

Schlüsselwörter Innerpsychischer Konflikt · Individuation · Abhängigkeit ·

Coachingbeziehung

Claudia Halberstadt $(\square)$

Institut für Psychologie, Fachgebiet Theorie und Methodik der Beratung, Universität Kassel,

Holländische Str. 36-38, 34127 Kassel, Deutschland

E-Mail: ch@claudia-halberstadt.de 


\title{
The inner conflict "individuation vs. dependency" in coaching: Key findings from a qualitative study on experienced coaches' practice
}

\begin{abstract}
Inner-psychic unconscious conflicts are an important part of a person's unique personality and they presumably have an impact on coaching processes. This study focusses on the "individuation vs. dependency conflict" in individual coaching and explores the practical knowledge of experienced professional coaches. In a small field study, 13 explorative semi-structured interviews were conducted with experienced coaching practitioners. The interviews focused on what appeared to be helpful in coaching. The qualitative content analysis delivered results that point to strategies in coaching. The article focuses primarily on the discussion of a central finding: the relevance of the working-alliance between coach and coachee.
\end{abstract}

Keywords Inner-psychic conflict · Individuation · Dependency · Working-alliance

\section{Einleitung}

Wenn Menschen Coaching in Anspruch nehmen, bringen sie existenzielle Lebensthemen und biografisches Gewordensein mit in die Beratungssituation. Dazu gehören auch innerpsychische Grundkonflikte. Dass diese einen Einfluss auf (arbeitsweltliche) Beratungsprozesse haben, kann vermutet werden, gesichertes Wissen dazu fehlt bislang. Mit Blick auf innerpsychische Konflikte von Coachees und ihr (gelingendes) Handling im Coaching - der Fokus dieser Studie - aber auch im Hinblick auf den Einfluss innerpsychischer Konflikte von Coaches - fehlt es bislang an Forschung. In der Coachingforschung gibt es bisher noch keine Einigkeit darüber, inwiefern die Persönlichkeit von Coachees und Coaches Einfluss auf die Coachingprozesse hat. Persönlichkeitsmerkmale, Motive und Haltungen gelten zwar als relevante Einflussvariablen auf der Inputseite (Ely et al. 2010; Greif et al. 2012; Behrend und Greif 2018), doch sind ihre Effekte meist schwächer als erwartet. Analysen zum Stand empirischer Coachingforschung verweisen darauf, dass zu Coachmerkmalen (u. a. Coachingexpertise) und dem Coacheemerkmal Persönlichkeit als Einflussgröße noch erheblicher Forschungsbedarf besteht (Kotte et al. 2016; Athanasopoulou und Dopson 2018). Insgesamt liegen zur Inputseite nur verhältnismäßig wenige Studien vor (vgl. Kotte et al. 2015; De Haan 2019; Grant und O’Connor 2019).

\section{State of the Art: Theoretische Hintergründe}

Die wissenschaftliche Rahmung der Studie bezog mehrere Stränge ein: Ein mehrperspektivischer Zugang zum Thema wurde gewählt, indem unterschiedliche Wissensbestände herangezogen, Ergebnisse qualitativer und quantitativer Forschung sowie das Praxis-Erfahrungswissen erfahrener Coaching-Expert/innen im Forschungsprozess miteinander kombiniert wurden. 


\subsection{Das Schlüsselkonzept: Der innerpsychische Konflikt}

Die Studie bezieht sich auf das theoretische Konflikt-Modell intrapsychischer bzw. innerseelischer Konflikt des Arbeitskreises Operationalisierte Psychodynamische Diagnostik (Arbeitskreis OPD 2006). Seine Besonderheiten und die Phänomenologie des ,Individuation vs. Abhängigkeit-Konflikts“ sollen im Folgenden kurz dargestellt werden. Als tiefenpsychologisch-psychodynamisch fundiertes diagnostisches Instrumentarium beschreibt die OPD intrapsychische Konflikte als generelle, fundamentale Dimensionen des Erlebens und Verhaltens von Menschen, die sich auf der Ebene des Individuums in typischen Selbst- und Weltbildern, Beziehungs- und Konfliktmustern und Affekten zeigen. Für die OPD ist die Vorstellung zentral, dass basale psychische Bedürfnisse des Menschen wie z. B. Autonomie und Bindung, Quellen ,,meist unbewusster innerseelischer Zusammenstöße entgegengesetzter Motivbündel“ sind. Aus ihnen entwickeln sich intrapsychische Konflikte (a.a.O., S. 96), mit denen ,festgelegte Erlebens- und Verhaltensmuster (...) verbunden sind (...), die in entsprechenden Situationen immer wieder aktiviert werden, ohne dass dies dem Menschen bewusst wäre und ohne dass er sie aus eigener Willensanstrengung überwinden könnte (neurotische Fixierung)“ (ebd.). Beschrieben wird, dass innerpsychische Konflikte zum Auslöser äußerer Konflikte werden und interpersonale Konfliktlagen innerpsychische Konflikte aktivieren können.

Lebensbestimmend wird ein innerpsychischer Konflikt, wenn die normale bi-polare Spannung eines Motivsystems in eine konflikthafte Polarisation übergegangen ist (a. a. O., 216f.) und sich infolgedessen dysfunktionale Lösungsmodi verfestigt haben; damit gewinnt das Konfliktthema eine existenzielle Bedeutung für die Person und prägt ihre Lebensgeschichte und Persönlichkeit. Von Integration als gelungener Bewältigung des Konflikts unterscheidet die OPD zwei prototypische dysfunktionale Bewältigungsformen, den aktiven und den passiven Lösungsmodus. In Abhängigkeit von weiteren psychodynamischen Variablen wie „Struktur“ und „Beziehungsmuster“ werden dysfunktionale Bewältigungsmuster als Quelle seelischer Belastungen und individueller psychischer Vulnerabilität verstanden, die sich in graduellen Abstufungen von mäßigen Symptomen bis hin zu einer leidvollen existenziellen seelischen Krise zeigen können.

\subsection{Zur Phänomenologie des Individuation vs. Abhängigkeit-Konflikts}

Jeder innerpsychische Konflikt zeigt sich nach dem theoretischen Modell der OPD phänomenologisch auf interpersonaler Ebene; für den Individuation vs. Abhängigkeit-Konflikt wird hervorgehoben, dass sich das dysfunktionale Konfliktmuster in der Außenwelt ganz besonders in den Dimensionen von Kontakt und Beziehung manifestiert.

Folgt man der OPD, besteht eine gelungene Entwicklung des Konfliktthemas darin, dass ein Mensch beide Motive psychisch integrieren kann, diese also nicht als Widerspruch erlebt. „Die Person kann enge, emotional nahe und tiefe Beziehungen eingehen (Abhängigkeit) und sich gleichzeitig als abgegrenztes, autonomes, eigenständiges Individuum erleben (Individuation), weder Alleinsein noch In-Beziehung-Sein löst (unbewusste) Ängste aus“ (Benecke und Möller 2013, S. 190). 
Für Coachees im ,aktiven Modus“ hingegen wird als typisch beschrieben, dass für sie die Wahrung der Unabhängigkeit von Beziehungen primäres Lebensziel ist, dass Angst vor Nähe und vor Bindung das Erleben von Beziehungen zu anderen prägt. Das Selbstbild der Person ist dominiert von Vorstellungen eigener Unabhängigkeit und Eigenständigkeit bzw. der Vorstellung, ,auf andere nicht-angewiesen zu sein“. Bedürfnisse nach Anlehnung und Nähe müssen abgewehrt werden.

Für Coachees mit einer Aktivierung im „passiven Modus“ ist charakteristisch, dass Vorstellungen von existenziellem Angewiesen-Sein auf emotionale Bindungen dominieren, nahe intime Beziehungen auch am Arbeitsplatz hergestellt werden, dass alles, was enge Beziehungen erschüttert, gemieden und Eigenständigkeit - auch die der anderen - zurückgewiesen, Trennungen von anderen verhindert werden müssen, Konflikte, Dissens, Konkurrenz ignoriert oder bagatellisiert werden. Zur Phänomenologie dieses Grundkonflikts gehören laut OPD spezifische Kontaktmuster sowie Verhaltens- und Erlebensweisen bezogen auf Nähe und Distanz in Beziehungen. Es erscheint sehr plausibel, dass diese Dispositionen den Beratungskontakt bzw. die Coachingbeziehung beeinflussen werden.

\subsection{Forschungsdesign, -prozess und Forschungsfrage}

Zur theoretischen Fundierung wurde ein Überblick über den ,state of the art“ zum Handling des Individuation vs. Abhängigkeit-Konflikts in etablierten, wissenschaftlich fundierten Ansätzen von Beratung/Counselling, Coaching und Psychotherapie erarbeitet. Dieser bildete die Ausgangsbasis für die eigene empirische Forschung. Forschungsannahmen wurden auf der Basis konkurrierender Leitaussagen etablierter theoretischer Denktraditionen (humanistische Psychologie: Gestalt und personenzentrierter Ansatz; psychodynamisch/psychoanalytischer Ansatz) in Bezug auf wissenschaftlich fundierte Coachingansätze sowie unter Berücksichtigung von Studienergebnissen aus qualitativ-quantitativer Coaching-, Psychotherapieforschung und aus Wirkfaktorenmodellen entwickelt.

Die Forschungsfrage lautete: Was zeigt sich aus Sicht erfahrener Coaches als hilfreich (oder weniger hilfreich) im Coaching von Coachees mit aktiviertem Grundkonflikt Individuation vs. Abhängigkeit? In der qualitativen Feldstudie $(n=13)$ wurden face-to-face explorative, teilstrukturierte Interviews mit erfahrenen CoachingExpert/innen durchgeführt. Sie wurden zu ihren subjektiven Theorien und präferierten Arbeitskonzepten im Handling des Individuation vs. Abhängigkeit-Konflikts im Einzelcoaching befragt. Das empirische Material wurde qualitativ ausgewertet. Die inhaltsanalytischen Ergebnisse sind in Form eines gemischt induktiv-deduktiv abgeleiteten Kategoriensystems systematisiert (Schreier 2012).

\section{Methodik, Datenerhebung und Sampling}

Im Interview wurde exploriert, ob die mit dem innerpsychischen Konflikt Individuation vs. Abhängigkeit verbundenen Erlebens- und Verhaltensweisen der Coachees im Coaching als Priorität aufgegriffen wurden, worin sich die Coachings von anderen Coachings unterschieden, wie unterschiedlich das Vorgehen im Bezug zum 
aktiven und passiven Lösungsmodus war und wie im Coaching in Phasen von Labilisierungen/Krisen des Coachee gehandelt wurde. Eine auf der Grundlage der OPD entwickelte Beschreibung modus-typischer Erlebens- und Verhaltensweisen von Personen mit aktiviertem Grundkonflikt Individuation vs. Abhängigkeit im aktiven Lösungsmodus (aMCoachee) und im passiven Lösungsmodus (pMCoachee) wurde den Interviewees zu Beginn des Interviews vorgelesen.

Die Datenerhebung fand im Zeitraum von Dezember 2019 bis März 2020 statt. Das Sampling berücksichtigte erfahrene Coaches und bezog unterschiedliche Ausbildungshintergründe und theoretische Zugehörigkeiten (psychodynamisch, psychoanalytisch; systemisch/hypno-systemisch; gestaltorientiert; personenzentriert) ein, die auf wissenschaftlich fundierte Coachingansätze eingegrenzt waren. 8 Expert/ innen gaben an weiblich, 5 Expert/innen gaben an männlich zu sein. 3 Expert/innen gaben ein Alter über 71 Jahren, 7 ein Alter zwischen 60 und 67 Jahren, 3 ein Alter zwischen 50 und 59 Jahren an. 11 der 13 Expert/innen gaben an, psychotherapeutisch ausgebildet zu sein, 8 davon gaben an, psychotherapeutisch zu praktizieren. Im Hinblick auf das Kriterium „Erfahrung“ orientierte sich das Sampling an der Forschungspraxis qualitativer Studien zu Selbstaussagen von Coaches bzw. Berater/ innen (z. B. De Haan et al. 2016). Eingeschlossen wurden Coaches mit a) mindestens 7 Jahren Berufserfahrung nach Abschluss einer einschlägigen Coachingausbildung und b) einem Praxisschwerpunkt im Bereich Einzelcoaching.

\section{Zentrale Studienergebnisse}

\subsection{Auf die Beratungsbeziehung kommt es an: Die Gestaltung der Working- Alliance als Schlüsselfaktor}

In Übereinstimmung mit der Literatur (Grawe 1998; Grawe et al. 1994; Dörrich 2017) kann die Beratungsbeziehung ausgehend von den inhaltsanalytischen Ergebnissen als Schlüsselfaktor im Handling des Individuation vs. Abhängigkeit-Konflikts in der Praxis erfahrener Coaches betrachtet werden. Erfahrene Praktiker/innen benennen die Gestaltung der Beratungsbeziehung, die gemeinsame Reflexion der Beziehungserfahrung und die Gestaltung des Kontakts entlang der Beziehungsthemen von Nähe und Distanz als besonders relevant und hilfreich. Eine gute Zusammenarbeit von Coach und Coachee gilt unabhängig vom theoretischen Hintergrund als Voraussetzung für erfolgreiches Coaching. Die Beratungsbeziehung wird als das Medium verstanden, in dem Coaching stattfindet. Stober und Grant (2009, S. 230) formulieren dazu: ,(...) regardless of the preferred theoretical background, the foundation of effective coaching is the successful formation of a collaborative relationship“. Etablierte Wirkfaktorenmodelle sehen die Beratungsbeziehung als basalen Faktor (vgl. Stober und Grant 2009; Greif et al. 2012; Ely et al. 2010). Empirische Studien zur Working-Alliance im Coaching zeigten, dass die Qualität der Arbeitsbeziehung stärker über ,experienced helpfulness“ entscheidet als andere Prozessfaktoren (vgl. De Haan et al. 2011).

Die explizite Bezugnahme auf die Beratungsbeziehung, wie z.B. das Thematisieren der Beziehungsdynamik, die Verständigung über Erfahrung und Kontakt in 
Tab. 1 Vier-Felder Matrix: Taxonomie des Beziehungsverhaltens der Coaches zum Individuation vs. Abhängigkeit-Konflikt im Coaching. (Eigene Darstellung)

\begin{tabular}{lll}
\hline $\begin{array}{l}\text { Beziehungsverhalten Coach: Kontaktgestaltung } \\
\text { Nähe-Distanz }\end{array}$ & $\begin{array}{l}\text { Subcodes } \\
\text { aktiver Modus } \\
n\end{array}$ & $\begin{array}{l}\text { Subcodes } \\
\text { passiver Modus } \\
n\end{array}$ \\
\hline $\begin{array}{l}\text { Kontrastierend-komplementär: } \\
\begin{array}{l}\text { Coachee-Muster durchbrechen } \\
\text { Responsiv: } \\
\text { Coachee-Muster aufnehmen/beachten }\end{array}\end{array}$ & 2 & 14 \\
\hline
\end{tabular}

$n$ : Codierungshäufigkeit

der Beratungsbeziehung gemeinsam mit Coachees sind jedoch auch Gegenstand theoretischer Diskussionen (vgl. Helbing 2014). Wenn es Coaches als hilfreich erscheint, Beratungskontakt und -beziehung explizit zum Thema zu machen, falls der innerpsychische Konflikt Individuation vs. Abhängigkeit beim Coachee vorliegt, könnte das auf ein Spezifikum seines Handlings im Coaching hinweisen, nämlich, dass es besonders bedeutsam ist Ergebnisse der Analyse von Gegenübertragungen zur Verfügung zu stellen, wenn der Konflikt aktiviert ist.

Was ist nun das Spezifische in der Gestaltung der Beratungsbeziehung mit Coachees mit dem aktivierten Grundkonflikt Individuation vs. Abhängigkeit? Die Analyse des empirischen Materials ergab ein Codierungsmuster, das zeigt, dass und wie erfahrene Coaches den aktiven Umgang mit Kontaktmustern von Coachees im aktiven und im passiven Modus als hilfreiches Instrument beschreiben. Auf Basis der empirischen Daten konnte ihr Beziehungsverhalten in einer Taxonomie geordnet werden. Sie beschreibt, wie erfahrene Coaches die Nähe- und Distanzregulation im Beratungskontakt in Relation zum Kontaktmuster des aktiven und des passiven Modus gestalten. Sie verfolgen Beziehungsstrategien, beantworten das Coachee-Kontaktmuster „responsiv“ oder „kontrastierend-komplementär“, nehmen es auf oder durchbrechen es (vgl. Tab. 1).

Im Coaching mit Coachees im aktiven Modus wird ein Beziehungsverhalten als hilfreich beschrieben, das die ausgeprägten Distanzbedürfnisse des Coachee einfühlend berücksichtigt. Dagegen wird im Coaching mit Coachees im passiven Modus als hilfreich berichtet, ausgeprägte gezeigte Nähe- und Bindungsbedürfnisse des Coachee als Gegenüber zurückzuweisen. Im Coaching mit Coachees mit aktiviertem Individuation vs. Abhängigkeit-Konflikt sollen auf diese Weise Nähe und Distanz in der Waage gehalten werden. Erfahrene Coaches nehmen an, dass das Ausbalancieren des Kontakts zu positiven Ergebnissen im Coaching führt.

Im Hinblick auf den aktiven Modus (Distanzbedürfnisse) zeigte sich das im Material z. B. konkret an Aussagen wie: „Und da weiß ich, dass sagen wir mal unsere (...) Arbeitsbeziehung, glaube ich, deswegen Bestand hat (...), weil ich mich auch ihm gegenüber distanziert genug verhalten habe. Also ich bin ihm nicht nachgelaufen. (...). Und ich habe ihm seinen Platz gelassen. Ohne (...) zu insistieren. Es geht also darum, zu schauen, womit rücke ich ihm zu nah auf die Pelle. (...) Also zu sehen, wichtig ist, dass er die nötige Distanz spürt" (Interviewpartnerin 10).

Bezogen auf die Beziehungsgestaltung mit Coachees im passiven Modus beschrieben Coaches folgendes Vorgehen: „Also den Abstand suchen, (...) den Ab- 
stand. Also das ist ja immer wichtig, den Abstand zu halten. Ist überhaupt das Allerwichtigste in Beratung. Aber bei den beziehungsorientierten Menschen ist es auch anstrengender, den Abstand zu halten. Und desto nötiger" (Interviewpartnerin 1).

Dieses Teilergebnis verdeutlicht, dass erfahrene Coaches der Regulation von Nähe und Distanz eine zentrale Bedeutung zuschreiben. Das Beziehungsverhalten wird bewusst als Instrument eingesetzt, um passgenau Veränderung zu unterstützen. Das Vorgehen setzt eine aufmerksame Beobachtung von Bindungs- und Autonomiebedürfnissen der Coachees im Beratungskontakt voraus.

Je nachdem, wie typische Verhaltensweisen und Neigungen des Coachee es aus Sicht des Coachs erforderlich machen, gestalten sie die Beziehung zum Coachee eher einfühlend-unterstützend oder eher konfrontierend-herausfordernd. Ausgehend von den Berichten scheint förderlich, die Beziehung zu Coachees im aktiven Modus einfühlend-unterstützend zu gestalten, sodass diese sich in ihrem Distanzbedürfnis als vom Gegenüber wahrgenommen erleben können. Coaches berichteten z. B. wie sie sich am Distanzwunsch angepasst, im Kontakt mit Bedacht zurückhaltend verhielten, oder ein eher kühles, wenig emotionales Mikroklima im Kontakt aufrechterhielten.

In Beziehung zu Coachees im passiven Modus scheint Coaches ein konfrontierend-herausforderndes Verhalten entwicklungsförderlich. Coaches drücken Dissens und Widerspruch direkt aus, sie muten Spannungen zu, sie konfrontieren Angepasstheit des Coachee, sie weisen privat-informelle Beziehungswünsche des Coachee offen zurück oder sie sorgen für längere zeitliche Distanz zwischen Beratungskontakten. Dass es für ein Coaching mit Coachees im passiven Modus als hilfreich berichtet wird, Spannung in der Beziehung zu generieren, um Distanz herzustellen, zeigte sich im Material an Äußerungen wie z. B.: ,(...) hilfreich ist, dass ich eine eigene und konträre Position einnehme, sodass vom Coachee ausgehalten werden muss, wir sind nicht einer Meinung (...), und erlebt wird, dass auch dann etwas Sinnvolles dabei herauskommt" (Interviewpartner 12). Wie intensivierte Nähebedürfnisse des Coachee im passiven Modus im Beratungskontakt zurückgewiesen und konfrontiert werden, zeigte sich an Äußerungen wie: „Die Leute versprechen sich privaten Kontakt. Irgendwann muss man dann Stellung beziehen und muss sagen: ,Wissen Sie, ich bin am hilfreichsten für Sie, wenn ich diesen Abstand haben kann. Wenn Sie mich jetzt nicht auf Ihre Grillparty einladen, (...) sondern wenn wir diese Situation hier gestalten (...), dass wir das spezielle Setting, das wir hier haben, bewahren ““ (Interviewpartnerin 13). Oder wie: ,(...) ich hab' das offen gemacht und ihm gesagt: ,Wir sind hier in einer professionellen Beziehung, da gebe ich keine privaten Statements ab, wir sind hier nicht - auch nicht demnächst - irgendwie miteinander befreundet"“ (Interviewpartnerin 4).

Im Hinblick auf das Coaching von Coachees im aktiven Modusstreben geben Coaches als zentral an, „Rapport“ sowie die Bereitschaft, sich auf Coaching einzulassen (commitment) zu stärken. Als wichtig erscheint es, dafür gerade am Beginn von Prozessen, Distanzwünsche des Coachee zu berücksichtigen. Dies zeigte sich im Material an Äußerungen wie: ,(...) er hat für das erste Coaching einen Hotelraum gewählt in dem wir (...) nicht völlig allein waren, so dass es halböffentlich war, was für das Kennenlernen dazu führte, dass ich auf Distanz bleiben musste und es auch nicht zu emotional werden konnte. Erst später konnte sich das ändern. Im 
Nachhinein stellte sich heraus, dass er das so inszeniert hat" (Interviewpartner 3). Oder wie: ,Wenn ich in den ersten Stunden merke, dass jemand immer wieder aus dem Kontakt rauszugehen versucht, wenn das für ihn zu dicht oder zu persönlich wird - auch wenn ich weiß, Coaching funktioniert so nicht, ohne Kontakt, dann thematisiere ich das aber bei diesem Typus grundsätzlich nicht, ich konfrontiere da nicht" (Interviewpartnerin 2).

In den subjektiven Theorien erfahrener Expert/innen wird damit den sogenannten „Bindungs- und Beziehungsaspekten der Working-Alliance“ (Bordin 1979) eine besondere Bedeutung für das Gelingen von Coachingprozessen zugeschrieben. Für Coaching mit Coachees im aktiven Modus zeigte sich das z. B. im Material bei Antworten auf die Frage, was das wichtigste Hilfreiche für diese Coachings sei an Äußerungen wie: ,(...) über das Coaching exemplarisch eine Erfahrung von Nähe aufbauen (...). Also von Nähe heißt an der Stelle bereit zu sein, (...) Emotionen mitzuteilen, seine Geschichte mitzuteilen“ (Interviewpartner 3).

\subsection{Auf die Arbeit an persönlichen Entwicklungsfeldern kommt es an: Veränderung im Coaching bei Coachees mit dem ,Individuation vs. Abhängigkeit-Konflikt"“}

Coachingspezifische Interventionen wurden für beide Konfliktlösungsmodi, also für den aktiven Modus (Individuations-Pol) und den passiven Modus (AbhängigkeitsPol) inhaltsanalytisch kategorisiert. Hier wurde einerseits eine Veränderung und Entwicklung der Coachees angeregt. Interventionen fokussierten die innere Haltung, die Wahrnehmung und darauf, Verhalten zu flexibilisieren. Andererseits wurde bei fehlender Passung von Person und Rolle/Funktion eine Veränderung des beruflichen Umfelds angestoßen und damit Veränderung im Außen angeregt. Erfahrene Coaches wählten beide Vorgehensweisen, doch blieb unklar, nach welchen Kriterien sie die Richtung der Intervention entschieden. Das sollte künftige Forschung untersuchen.

Im Hinblick auf die Forschungsfrage zeigte es sich für beide Lösungsmodi als hilfreich, einen Interventionsfokus auf der Ebene der Person zu setzen und die Bearbeitung persönlicher Themen explizit mit zu kontraktieren oder sie nachträglich in den Kontrakt aufzunehmen.

Für beide Konfliktlösungsmodi wurde ein Querschnittsthema identifiziert: Erfahrene Coaches gaben der Reflexion der „Passung von Person und Rolle/Funktion“ hohe Priorität, sie unterstützten Coachees im aktiven wie im passiven Modus darin, die Stimmigkeit von Zielen und Aufgaben, beruflichen Positionen oder professionellen Rollen im Hinblick auf diesen Anteil der Persönlichkeit - die als existenziell erlebten Autonomie- und Distanz- bzw. Nähebedürfnisse - zu überprüfen, ihre Bedürfnisse zu respektieren und im Coaching selbst-kongruente Ziele und Selbstakzeptanz zu entwickeln. Als hilfreich wurde berichtet, Coachees anzuregen, sich selbst intensiver wahrzunehmen (awareness) und zu verstehen. Im Material zeigte sich das an Beispielen wie „Im Coaching sollen die Leute verstehen - aha, also ich bin so, und andere sind da ganz anders" (Interviewpartnerin 1).

Wenn Coachees aufgrund des innerpsychischen Konflikts sogenannte dysfunktionale Führungsbeziehungen zu Mitarbeiter/innen entwickelt haben, wurde als hilfreich beschrieben, das Phänomen explizit zu konfrontieren. Dazu fanden sich für 
Tab. 2 Handling des Individuation vs. Abhängigkeit-Konflikts im Coaching - Beratungspraktische Implikationen inhaltsanalytischer Befunde. (Eigene Darstellung)

\begin{tabular}{ll}
\hline Handling des „Individuation vs. Abhängigkeit-Konflikts“ & $\begin{array}{l}\text { State of the Art der Litera- } \\
\text { tur: } \\
\text { Referenztheorien und } \\
\text { Kerndimensionen von }\end{array}$ \\
$\begin{array}{l}\text { Veränderung bei ,Indi- } \\
\text { viduation vs. Abhängigkeit- } \\
\text { Konflikt }^{“ 4}\end{array}$ \\
\hline
\end{tabular}

Selbstreflexion: Innerpsychischer Konflikt als Quelle äußerer Konflikte

- Bewusstmachung des eigenen Modus: am Verständnis inter-personeller Dynamik arbeiten, Kontaktverhalten des Coachee in Arbeitsbeziehungen reflektieren, Perspektivwechsel anregen (,Wie wirke ich auf andere?")

- autobiografische Selbsterkundungen anregen

- Einsicht fördern, dass Individuation/Abhängigkeit bzw. Nähe-Distanz-Bedürfnisse im Arbeitskontext nur relativ eingeschränkt gelebt werden können

- verstehen, dass dysfunktionale Führungs- und Arbeitsbeziehungen mit dem jeweiligen Lösungsmodus (aktiv: Individuations-Pol; passiv: Abhängigkeits-Pol) zusammenhängen. kritische Auseinandersetzung mit zu großer Nähe und freundschaftlich-intimen Beziehungen mit Teammitgliedern (passiver Modus) bzw. mit zu großer Distanziertheit (aktiver Modus) im Coaching

\begin{tabular}{|c|c|}
\hline $\begin{array}{l}\text { Selbstakzeptanz und -verantwortung bzgl. eigener Bedürfnisse } \\
\text { - Bedürfnisse von Autonomie/Bindung bzw. Nähe/Distanz in Bezie- } \\
\text { hungen als wichtig anerkennen, im Coaching daran arbeiten, wie } \\
\text { diese Bedürfnisse außerhalb des Arbeitskontexts gelebt werden } \\
\text { können; Lebensbereiche kultivieren, in denen diese gelebt werden } \\
\text { können; dazu innere und äussere Ressourcen aktivieren; Laufbahn- } \\
\text { entscheidung prüfen } \\
\text { - Coachees im passiven Modus: Unterstützen, Privatheit und } \\
\text { Nicht-Privatheit von Beziehungen in Freundschaft und in Arbeitsbe- } \\
\text { ziehungen zu trennen } \\
\text { - Coachees im aktiven Modus: Lebensbereiche, in denen Bedürfnis- } \\
\text { se nach Eigenständigkeit/Unabhängigkeit gelebt werden können, } \\
\text { identifizieren, anregen diese zu kultivieren }\end{array}$ & $\begin{array}{l}\text { Selbstakzeptanz, Selbstak- } \\
\text { tualisierung, Selbstempa- } \\
\text { thie, Selbstverantwortung } \\
\text { Rogers (1987); Hutterer } \\
\text { (1992, 2009); Perls et al. } \\
\text { (1951); Benecke und Möller } \\
\text { (2013); Bachkirova (2011); } \\
\text { Stober (2009); Kriz (2009); } \\
\text { Höll (2009) }\end{array}$ \\
\hline $\begin{array}{l}\text { Stärken der Fähigkeit zur Affektregulation und Fähigkeit zum } \\
\text { Umgang mit als unangenehm erlebten Emotionen stärken } \\
\text { - Coachees im passsiven Modus: Angst vor Einsamkeit, Ärger, Dis- } \\
\text { tanz } \\
\text { - Coachees im aktiven Modus: Angst vor Kontakt, Nähe }\end{array}$ & $\begin{array}{l}\text { Umgang mit Emotionen, } \\
\text { Affektwahrnehmung und } \\
\text {-regulation } \\
\text { Arbeitskreis OPD (2006); } \\
\text { Greif et al. (2012); Bennett } \\
\text { (2003); Benecke und Möller } \\
\text { (2013) }\end{array}$ \\
\hline
\end{tabular}

\section{Einsicht, Bewusstheit,} Selbstreflexion

Benecke und Möller (2013); Grant et al. (2002); Greif und Rauen (2018); Bachkirova (2011)

Coachees im aktiven Lösungsmodus Beispiele wie: „Dann sag ich auch schon mal, also wenn Sie diese Dinge nicht aus der Hand geben können, weil Sie von ihren Mitarbeitern nicht abhängig sein wollen, dann machen Sie ihren Job als Führungskraft nicht" (Interviewpartner 12). Hilfreich fanden Expert/innen, Coaching in den Dienst der Entwicklung von Selbstreflexion und -kongruenz zu stellen und mit Coachees Laufbahnentscheidungen zu überprüfen. Für Coachees mit Aktivierung im passiven Modus gab es dazu Beispiele wie: „Wir sind dann dahin gekommen, dass sie (...) 
Tab. 2 (Fortsetzung)

Handling des „Individuation vs. Abhängigkeit-Konflikts“
State of the Art der Litera-

tur:

Referenztheorien und

Kerndimensionen von

Veränderung bei ,Indi-

viduation vs. Abhängigkeit-

Konflikt"“

Flexibilisieren des Coachee-Verhaltens

- Entwicklung von Strategien, um neues Verhalten in Beziehungen und der Gestaltung von Kontakt zu anderen im Arbeitskontext zu erproben: Flexibilisierung in Richtung des jeweils gegenüberliegenden Modus

Integration, Mittlerer Modus

Arbeitskreis OPD (2006);

Polster (2002); Perls et al. (1951)

Kontakt, Interesse für andere, Empathie Fuhr (2009); Perls et al. (1951); Polster (2002)

- Wahrnehmen von Nähe-/Distanzbedürfnissen anderer (Mitarbeiter/ innen, Teammitgliedern, Kund/innen)

- Ausbalancieren von Nähe und Distanz in Arbeitsbeziehungen

- Coachees im passiven Modus: Stärken von Konflikttoleranz, konfrontativer sein

- Coachees im aktiven Modus: Fähigkeit, Interesse für Mitarbeitende zu entwickeln, Fördern von Empathie für andere, ,,sich für Kontakt interessieren lernen"

\begin{tabular}{|c|c|}
\hline $\begin{array}{l}\text { Passung von Person und Rolle reflektieren - Kongruente Ziele } \\
\text { entwickeln } \\
\text { - Differenzieren: „Wenn ich so bindungs-/autonomieorientiert bin, } \\
\quad \text { welche Position/Aufgabe passt zu mir bzw. passt nicht zu mir?“” } \\
\text { - Veränderungen im Außen anstreben: Kontext/Umfeld wechseln }\end{array}$ & $\begin{array}{l}\text { Kongruenzerfahrungen } \\
\text { Selbstkongruenz } \\
\text { Stober (2009); Bachkirova } \\
(2004,2011) \text {; Greif et al. } \\
(2012)\end{array}$ \\
\hline $\begin{array}{l}\text { Gestaltung von Kontakt in der Arbeitsbeziehung (vgl. ausführliche- } \\
\text { re Beschreibung in 4.1) } \\
\text { - Coachees im passiven Modus: kontrastierend-komplementär, d.h. } \\
\text { Coachee-Kontakt-Muster durchbrechen (z. B. durch distanzierteres } \\
\text { und konfrontatives Beziehungsverhalten) } \\
\text { - Coachees im aktiven Modus: responsiv, d.h. Coachee-Kontakt-Mus- } \\
\text { ter: aufnehmen/beachten (z. B. durch mehr Zurückhaltung und Dis- } \\
\text { tanzierung) }\end{array}$ & $\begin{array}{l}\text { Coach-Coachee-Beziehung } \\
\text { (vgl. 4.1) }\end{array}$ \\
\hline $\begin{array}{l}\text { Unterstützung bei Krisen/Labilisierung } \\
\text { - Stärken der Ich-Instanz } \\
\text { - Stabilisierende Interventionen } \\
\text { - Containment/Holding }\end{array}$ & $\begin{array}{l}\text { Krise - Phasen von Labili- } \\
\text { sierung } \\
\text { Bion (1962); Winnicott } \\
(1958)\end{array}$ \\
\hline
\end{tabular}


ihre Fachkarriere wieder aufnimmt und die Führungsposition aufgibt (...), weil sie es als so tief schwierig erlebt hat, mit Konflikten umzugehen“ (Interviewpartner 11). Für Coachees mit Aktivierung im aktiven Modus beschrieben die Coaches etwa: „Wenn einer Autonomie so stark braucht und in Organisationen aber sehr viel in Kontakt sein, sich abstimmen muss, dann ist es einfach nicht das richtige ökologische Umfeld. Da berät man Menschen besser aus Organisationen hinaus“ (Interviewpartner 7). Tab. 2 zeigt beratungspraktische Implikationen der qualitativen Ergebnisse.

Das Vorgehen erfahrener Coaches entspricht theoretisch postulierten, durch die Psychotherapie-Forschung teilweise empirisch validierten Annahmen dazu, wie positive Veränderung und Entwicklung von Klient/innen unterstützt werden kann, ist aber für arbeitsweltliche Beratung, insbesondere für Coaching, so nicht ohne Weiteres erwartbar. Folgt man den Erfahrungswerten erfahrener Coaches (von denen jedoch eine Mehrheit einen psychotherapeutischen Hintergrund hat, vgl. Limitationen der Studie in 5.1) scheint eher hilfreich zu sein, wenn die Ebene der Person (Motivation, Werte, Bedürfnisse, Persönlichkeit) bei diesen Coachees fokussiert wird. Die Ebene der Interventionen, die sich auf Arbeit, Aufgaben, Rolle, Zuständigkeiten richtet, scheint im Coaching mit Blick auf den innerpsychischen Konflikt Individuation vs. Abhängigkeit in der Praxis weniger im Vordergrund zu sein.

Diese Ergebnisse der Analyse von Best Practices erfahrener Coaches haben beratungspraktische und -theoretische Implikationen. Sie verweisen darauf, dass bei innerpsychischen Konflikten im Coaching in der Praxis fließendere Übergänge zwischen den Beratungsformaten Coaching, Beratung und psychotherapeutischen Arbeitsweisen bestehen könnten, und - vorausgesetzt Coaches verfügen über psychotherapeutische Kompetenzen - dass es im Coaching sinnvoll sein kann, den Konflikt ,therapeutisch“ anzusprechen. Das wird besonders deutlich in Aussagen zum Umgang mit Krisen und mit Phasen psychischer Labilisierung von Coachees mit dem aktivierten Grundkonflikt Individuation vs. Abhängigkeit. Die Bewertung zur Verallgemeinerbarkeit des Ergebnisses müsste jedoch verzerrende Effekte, die sich aus der Besonderheit des Samples ergeben, berücksichtigen (s. oben).

\subsection{Auf innere Arbeit und externe Begleitung der Coaches kommt es an}

In Übereinstimmung mit der Literatur (vgl. z. B. Heuft 1990; Yontef 1999; Swanson und Lichtenberg 1998; Dreitzel 2004; Giernalczyk et al. 2012; Giernalczyk et al. 2013; Bachkirova 2016; Stober und Grant 2009) zeigte die inhaltsanalytische Auswertung für das Handling des aktivierten Grundkonflikts Individuation vs. Abhängigkeit im Coaching, dass erfahrene Coaches Selbstklärungsprozesse (Selbstreflexion, innere Arbeit), Intervision und Supervision als besonders hilfreich berichten, um sich in der Beziehung zu Coachees nicht zu verstricken. Das verweist möglicherweise auf besondere Herausforderungen im Umgang mit Übertragungen, die bestehen, weil sich dieser Grundkonflikt - vielleicht unmittelbarer als andere innerpsychische Konflikte der Coachees - in der Beratungsbeziehung manifestiert.

Das qualitative Ergebnis deutet auf hilfreiche Wirkungen von Intervision, Supervision für den Coaching-Outcome bei einem aktivierten Individuation vs. Abhängigkeit-Konflikt der Coachees hin. Das sollte Forschung weiter untersuchen. 


\section{Limitationen und Implikationen für künftige Coachingforschung und Coachingausbildung}

Die Forschungsergebnisse sind aus mehreren Gründen nicht ohne weiteres verallgemeinerbar. Einschränkungen ergeben sich insbesondere aus der Zusammensetzung des Samples, da die Mehrheit der Coaching-Expert/innen einen explizit psychotherapeutischen Background hatte und da ein Schwerpunkt ihrer Berufspraxis im Nonprofit-Bereich lag. Inwieweit die Arbeit an und mit der Coaching-Beziehung auch für Coaches ohne psychotherapeutische Ausbildung möglich und sinnvoll ist, ist daher (noch) nicht zu beantworten. Künftige Forschung sollte dieser Frage weiter nachgehen.

Künftige Forschung sollte außerdem überprüfen, ob aus der Perspektive anderer als der Coaches (insbesondere von Coachees, aber auch von Dritten, wie z.B. relevanten beruflichen Interaktionspartner/innen), ,erfahrenes Hilfreiches“ (experienced helpfulness) bestätigt wird. Daher sollte weitere Forschung Coaching-Dyaden fokussieren und sich auf die Mikroebene des Prozesses richten. Besonders interessant erscheint hier, mittels Beobachtungs-Methoden Coachverhalten und Interaktionen von Coachee und Coach auf der Ebene einzelner Sitzungen zu untersuchen.

Die Studienergebnisse implizieren, dass erfolgreiches Vorgehen im Coaching von Coachees mit dem innerpsychischen Konflikt Individuation vs. Abhängigkeit von Kompetenzen der Coaches abhängt, die diagnostische, interpersonelle und selbstreflexive Fähigkeiten umfassen. Das Handling des innerpsychischen Konflikts erfordert auch Professionalität im Umgang mit Phasen erhöhter emotionaler Spannungen und Krisen von Coachees und birgt besondere Herausforderungen, ein hilfreiches $\mathrm{Maß}$ an Nähe und Distanz in der Beratungsbeziehung auszubalancieren.

Auch wenn bezweifelt wird, dass nicht-tiefenpsychologisch ausgebildete Coaches psychoanalytisch-psychodynamische Interventionen ausreichend fachgerecht ausführen können (vgl. Grimmer und Neukom 2009; Kretschmar und Hamburger 2019), sollten Ausbildungscurricula daher z. B. auch diagnostisches Wissen aus tiefenpsychologischen Ansätzen berücksichtigen, um angehende Coaches für Grundkonflikte und deren mögliche Auswirkungen auf die Beratungsbeziehung zu sensibilisieren. Die Entwicklung von Selbstreflexions- und von Kriseninterventionskompetenz sollte etabliert sein, was für ausreichend lange Ausbildungszeiten spricht, die Coaches eine Entwicklung dieser Kompetenzen ermöglichen.

\section{Fazit}

Die qualitative Studie zum Handling des Individuation vs. Abhängigkeit-Konflikts im Coaching führte zur Entwicklung eines Kategoriensystems, das als orientierender Rahmen für den Umgang von Coaches mit diesem innerpsychischen Konflikt von Coachees genutzt werden kann. Die Ergebnisse verdeutlichen die Bedeutung von Coachee-Merkmalen und betonen die Bedeutung des Wirkfaktors Beratungsbeziehung. Gelingensfaktoren im Coaching von Coachees mit aktiviertem Individuation vs. Abhängigkeit-Konflikt liegen bei der Gestaltung des Kontakts: In der Beratungsbeziehung sollten Besonderheiten der Kontaktbedürfnisse von Coachees mit 
aktivem und mit passivem Modus differenziert beachtet werden. Für den Prozessfaktor Coach zeigt sich der Mehrbedarf an Selbstreflexion und Supervision. Als hilfreich berichtete Coachinginterventionen zum Individuation vs. Abhängigkeit-Konflikt (z. B. Selbstexploration und Selbstempathie anregen; Selbstakzeptanz, Einsicht/ Bewusstheit und Reflexion von Beziehungsverhalten fördern; Kriseninterventionen/ Einsatz stabilisierender Verfahren) stimmen mit der Praxis in benachbarten Arbeitsfeldern Counselling und Psychotherapie überein. Inwieweit dieses Vorgehen sich über die subjektive Einschätzung erfahrener Coaches hinaus als hilfreich erweist, muss sich in weiterer Forschung zeigen.

Funding Open Access funding enabled and organized by Projekt DEAL.

Open Access Dieser Artikel wird unter der Creative Commons Namensnennung 4.0 International Lizenz veröffentlicht, welche die Nutzung, Vervielfältigung, Bearbeitung, Verbreitung und Wiedergabe in jeglichem Medium und Format erlaubt, sofern Sie den/die ursprünglichen Autor(en) und die Quelle ordnungsgemäß nennen, einen Link zur Creative Commons Lizenz beifügen und angeben, ob Änderungen vorgenommen wurden.

Die in diesem Artikel enthaltenen Bilder und sonstiges Drittmaterial unterliegen ebenfalls der genannten Creative Commons Lizenz, sofern sich aus der Abbildungslegende nichts anderes ergibt. Sofern das betreffende Material nicht unter der genannten Creative Commons Lizenz steht und die betreffende Handlung nicht nach gesetzlichen Vorschriften erlaubt ist, ist für die oben aufgeführten Weiterverwendungen des Materials die Einwilligung des jeweiligen Rechteinhabers einzuholen.

Weitere Details zur Lizenz entnehmen Sie bitte der Lizenzinformation auf http://creativecommons.org/ licenses/by/4.0/deed.de.

\section{Literatur}

Athanasopoulou, A., \& Dopson, S. (2018). A systematic review of the executive coaching outcomes: is it the journey or the destination that matters the most? The Leadership Quarterly, 29(1), 70-88.

Arbeitskreis OPD (Hrsg.). (2006). Operationalisierte Psychodynamische Diagnostik OPD-2. Das Manual für Diagnostik und Therapieplanung. Bern: Hogrefe.

Bachkirova, T. (2004). Dealing with issues of self-concept and self-improvement. International Journal of Evidence Based Coaching and Mentoring, 2(2), 29.

Bachkirova, T. (2011). Developmental coaching. Developing the self. In J. Passmore, D. Peterson \& T. Freire (Hrsg.), Handbook of the psychology of coaching and mentoring (S. 135-154). London: Wiley.

Bachkirova, T. (2016). The Self of the Coach: Conceptualization, Issues and Opportunities for Practitioner Development. Consulting Psychology Journal: Practice and Research, 68(2), 143-156.

Behrend, P., \& Greif, S. (2018). Erfolgsfaktoren im Coaching. In S. Greif, H. Möller \& W. Scholl (Hrsg.), Handbuch Schlüsselkonzepte im Coaching (S. 163-172). Heidelberg: Springer. https://doi.org/10. 1007/978-3-662-45119-9_81-1.

Benecke, C., \& Möller, H. (2013). OPD-basierte Diagnostik im Coaching. In H. Möller \& S. Kotte (Hrsg.), Diagnostik im Coaching. Grundlagen, Analyseebenen, Praxisbeispiele (S. 183-198). Heidelberg: Springer.

Bennett, B. (2003). Developmental coaching: rejecting the remedial approach. Development and Learning in Organizations, 17(4), 16-19.

Bion, W. (1962). Learning from experience. London: Karnac Books.

Bordin, E.S. (1979). The generalizability of the psychoanalytic concept of the Working Alliance. Psychotherapy. Theory, Research \& Practice, 16, 252-260.

De Haan, E. (2019). A systematic review of qualitative studies in workplace and executive coaching: the emergence of a body of research. Consulting Psychology Journal: Practice and Research, 71(4), 227-248. https://doi.org/10.1037/cpb0000144. 
De Haan, E., Culpin, V., \& Curd, J. (2011). Executive coaching in practice: What determines helpfulness for clients of coaching? Personnel Review, 40(1), 24-44. https://doi.org/10.1108/00483481111095500.

De Haan, E., Burger, Y., \& Grant, A. M. (2016). A large scale study of executive and workplace coaching: the relative contributions of relationship, personality match, and self-efficacy. Consulting Psychology Journal: Practice and Research, 68(3), 189-207.

Dörrich, V. (2017). Die Übertragbarkeit der Wirkfaktoren der Psychotherapie nach Grawe et al. auf die Beratung. https://digibib.hs-nb.de/file/dbhsnb_thesis_0000001677/dbhsnb_derivate_0000002361/ Masterthesis-Doerrich-2017.pdf. Zugegriffen: 1. Sept. 2021.

Dreitzel, P. (2004). Gestalt und Prozess - eine psychotherapeutische Diagnostik. Oder Der gesunde Mensch hat wenig Charakter. Köln: EHP.

Ely, K., Boyce, L.A., Nelson, J.K., Hernez-Broom, G., \& Whyman, W. (2010). Evaluating leadership coaching. A review and integrated framework. The Leadership Quarterly, 21(4), 585-599. https:// doi.org/10.1177/0021886315576407.

Fuhr, R. (2009). Kontakt. In G. Stumm \& A. Pritz (Hrsg.), Wörterbuch der Psychotherapie (2. Aufl. S. 364). Heidelberg: Springer.

Giernalczyk, T., Lazar, R., \& Albrecht, C. (2012). Die Rolle der Führungskraft und des Beraters als Container. In T. Giernalczyk \& M. Lohmer (Hrsg.), Das Unbewusste im Unternehmen: Psychodynamik von Führung, Beratung und Change-Management (S. 45). Stuttgart: Schäffer-Poeschel.

Giernalczyk, T., Lohmer, M., \& Albrecht, C. (2013). Containment im coaching. Organisationsberatung, Supervision, Coaching, 20(4), 425-435.

Grant, A.M., \& O'Connor, S. (2019). A brief primer for those new to coaching research and evidencebased practice. The Coaching Psychologist, 15(1), 2019.

Grant, A., Franklin, J., \& Langford, P. (2002). The self-reflection and insight scale: a new measure of private self-consciousness. Social Behavior and Personality, 30(8), 821-835. https://doi.org/10.2224/ sbp.2002.30.8.821.

Grawe, K. (1998). Psychologische Therapie. Göttingen: Hogrefe.

Grawe, K., Donati, R., \& Bernauer, T. (1994). Psychotherapie im Wandel: Von der Konfession zur Profession (3. Aufl.). Göttingen: Hogrefe.

Greif, S., \& Rauen, C. (2018). Selbstreflexion im Coaching. In S. Greif, H. Möller \& W. Scholl (Hrsg.), Handbuch Schlüsselkonzepte im Coaching (S. 523-531). Heidelberg: Springer.

Greif, S., Schmidt, A., \& Thamm, F. (2012). Warum und wodurch Coaching wirkt. Ein Überblick zum Stand der Theorieentwicklung und Forschung über Wirkfaktoren. Organisationsberatung, Supervision, Coaching, 19(4), 375-390. https://doi.org/10.1007/s11613-012-0299-4.

Grimmer, B., \& Neukom, M. (2009). Coaching und Psychotherapie. Gemeinsamkeiten und Unterschiede - Abgrenzung oder Integration. Wiesbaden: VS.

Helbing, M. (2014). Über die Bedeutung der Beziehung im Coaching. OSC, 21(1), 115-124.

Heuft, G. (1990). Bedarf es eines Konzeptes der Eigenübertragung? Forum der Psychoanalyse, 6(4), 299-315.

Höll, K. (2009). Selbstverantwortung. In G. Stumm \& A. Pritz (Hrsg.), Wörterbuch der Psychotherapie (2. Aufl. S. 634). Heidelberg: Springer.

Hutterer, R. (1992). Aktualisierungstendenz und Selbstaktualisierung. Eine personenzentrierte Theorie der Motivation. In R. Hutterer \& R. Stipsitis (Hrsg.), Perspektiven rogerianischer Psychotherapie (S. 146-171). Wien: WUV.

Hutterer, R. (2009). Selbstexploration. In G. Stumm \& A. Pritz (Hrsg.), Wörterbuch der Psychotherapie (2. Aufl. S. 627). Heidelberg: Springer.

Kotte, S., Oellerich, K., Schubert, D., \& Möller, H. (2015). Das ambivalente Verhältnis von Coachingforschung und Coachingpraxis. Dezentes Ignorieren, kritisches Beäugen oder kooperatives Miteinander? In A. Schreyögg \& C. Schmidt-Lellek (Hrsg.), Die Professionalisierung von Coaching. Ein Lesebuch für den Coach (S. 23). Wiesbaden: Springer.

Kotte, S., Hinn, D., Oellerich, K., \& Möller, H. (2016). Der Stand der Coachingforschung. Kernergebnisse der vorliegenden Metaanalysen. Organisationsberatung Supervision Coaching, 23(1), 5-23.

Kretschmar, T., \& Hamburger, A. (2019). Coaching und Supervision. Psychodynamische Beratung von Führungskräften. Stuttgart: Kohlhammer.

Kriz, J. (2009). Selbstaktualisierungstendenzen. In G. Stumm \& A. Pritz (Hrsg.), Wörterbuch der Psychotherapie (2. Aufl. S. 622). Heidelberg: Springer.

Perls, F. S., Hefferline, R., \& Goodman, P. (1951). Gestalt therapy. New York: The Julian Press.

Polster, E. (2002). Das Selbst in Aktion. In E. Polster \& M. Polster (Hrsg.), Das Herz der Gestalttherapie. Köln: EHP. 
Rogers, C. R. (1987). Eine Theorie der Psychotherapie der Persönlichkeit und der zwischen menschlichen Beziehungen. Köln: GwG.

Schreier, M. (2012). Qualitative content analysis in practice. Los Angeles: SAGE.

Stober, D. (2009). Coaching from a humanistic perspective. In D. Stober \& A. M. Grant (Hrsg.), The evidence based coaching-handbook (S. 29). San Francisco: Wiley.

Stober, D., \& Grant, A. M. (Hrsg.). (2009). The evidence based coaching-handbook. San Francisco: Wiley. Swanson, C., \& Lichtenberg, P. (1998). Diagnosis in gestalt therapy-a modest beginning. The Gestalt Journal, 21(1), 5-17.

Winnicott, D. W. (1958). Collected papers. Through paediatrics to psycho-analysis. London: Tavistock. Yontef, G. (1999). Awareness, Dialog, Prozess. Wege zu einer relationalen Gestalttherapie. Köln: EHP.

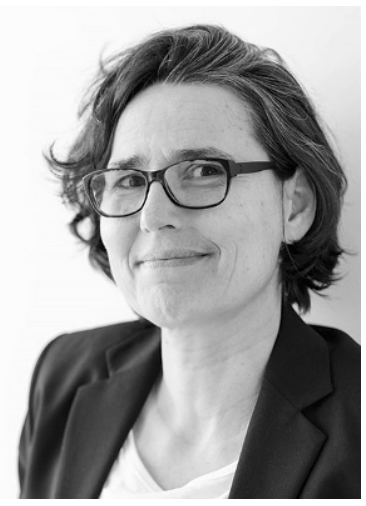

Claudia Halberstadt Profilangaben: Jg. 1963, Studium Bildende Kunst, Human-/Erziehungswissenschaften (Universität Heidelberg, Universität zu Köln); Beratungswissenschaften postgrad.M.A.COS (Universität Kassel); selbstständig; arbeitet in eigener Praxis als Coach, Supervisorin (DGSv), Organisationsberaterin und als freiberufliche Trainerin/Dozentin; tätig für profit/non-profit Organisationen; Malerin (BBK); weitere Informationen: www.claudia-halberstadt.de 